\title{
Analysis of Deficit Round Robin Scheduling for Future Aeronautical Data Link
}

\author{
Serkan Ayaz*, Felix Hoffmann*, Reinhard German ${ }^{\dagger}$ and Falko Dressler ${ }^{\ddagger}$ \\ ${ }^{*}$ German Aerospace Center (DLR), Institute of Communications and Navigation, Wessling, Germany \\ ${ }^{\dagger}$ Computer Networks and Communication Systems, Dept. of Computer Science, University of Erlangen, Germany \\ ${ }^{\ddagger}$ Institute of Computer Science, University of Innsbruck, Austria \\ \{serkan.ayaz,felix.hoffmann\}@dlr.de, german@cs.fau.de, dressler@ieee.org
}

\begin{abstract}
The aviation community is currently working on the standardization of data communication systems for the future air traffic management. In this context, the International Civil Aviation Organization (ICAO) has initiated a work on standardization of an IPv6-based aeronautical telecommunications network and on future radio access technologies, respectively. In this paper, we integrate L-Band Digital Aeronautical Communications System Option 1 (L-DACS 1), which is one candidate for future radio access technologies, with realistic IPv6-based network layer functionality and analyze Deficit Round Robin (DRR) with fragmentation algorithm for the forward and return link in L-DACS 1. Our analysis mainly covers two application domains: file transfer and real-time services. We show that DRR with fragmentation scheduler provides good performance results in terms of throughput, delay, and bandwidth fairness.
\end{abstract}

\section{INTRODUCTION}

In Air Traffic Management (ATM), there are two main communication services: Air Traffic Services (ATS) and Airline Operations Services (AOS) [1]. ATS Correspondent Nodes (CNs) are used to provide navigation, control, and situational awareness services to the aircraft (abbreviated as " $\mathrm{a} / \mathrm{c}$ " in the following), whereas AOS CNs are mainly used for business operations of airline companies. Using today's communication technologies, these services are generally performed by using analogue voice communications. However, digital data communication utilizes the bandwidth more efficiently and overall is much less error-prone. In addition, only with the the help of digital data communications, new ATM concepts like 4D trajectory exchange, graphical weather information transmission, and Voice over IP (VoIP) become possible.

For this reason, two main activities are running in parallel in order to build a future aeronautical communications network. The International Civil Aviation Organization (ICAO) is working on the one side on the standardization of the next generation IPv6-based Aeronautical Telecommunications Network (ATN/IPS) [2] and, on the other side, on the standardization of future radio access technologies for aeronautics.

In this work, we study the performance of scheduling algorithms for possible use in the aeronautical domain. In particular, we analyze Deficit Round Robin (DRR) with fragmentation for L-Band Digital Aeronautical Communications System Option 1 (L-DACS 1) integrated with the IPv6 network layer functionality. Our evaluation mainly covers file transfer and real-time services.

\section{A. Related Work}

With the advance of new wireless technologies (e.g., IEEE 802.16 and 3GPP LTE) that provide high data rates with different Class of Service (CoS) options and different application opportunities for mobile users, the design of appropriate scheduling algorithms become one of the most interesting topics in wireless communications. In this paper, we mainly consider the work done for IEEE 802.16 and for the LTE standards, because L-DACS 1 fundamentally also relies on Orthogonal Frequency Division Multiplexing (OFDM) concept.

In general, scheduling algorithms can be classified as channel aware and channel unaware [3]. It is also possible to subgroup channel unaware algorithms into intra-class, e.g., DRR [4] and Worst-case Fair Weighted Fair Queueing $\left(W F^{2} Q\right)$ [5], and inter-class, e.g., priority-based algorithms. While the former tries to solve the scheduling algorithms inside one class of service, the latter deals with scheduling of different service classes. In this paper, we focus on intra-class channelunaware resource allocation strategies and mainly on DRR with fragmentation due to its perfect throughput fairness with support of variable length packets together with a rather simple implementation [4], [6], [7]. In addition, as mentioned in [4], DRR can exhibit $O(1)$ processing work per packet. Since the wireless channel conditions in aeronautical case (especially in En Route and Terminal Maneuvering Area regions) are not changing abruptly [8] as opposed to consumer electronics domain (indoor, urban, etc.), we do not see a need to consider channel-aware scheduling algorithms.

From the applications perspective, three main application types are foreseen in the future ATM, namely file transfer, VoIP, and short message transfer applications. In this paper, we mainly consider file transfer of graphical weather information (WXGRAPH) on the Forward Link (FL) [9] and VoIP traffic between ATS controller and pilot. In [10], one of the initial studies regarding the usage of TCP in the future ATM has been presented. Furthermore, initial steps towards the usage of VoIP service have been carried out in [11].

\section{B. Contribution}

Our main contributions are the development and a thorough performance analysis of a scheduling architecture for L-DACS 1 . We design the scheduling block of L-DACS 1 in a hierarchical structure similar to [12]. While strict priority 
scheduling mechanism is working at the first level, different scheduling algorithms may be used for each service class at the second level. One disadvantage of the strict priority scheduling is that higher priority packets can get the most of the bandwidth so that lower priority packets can wait longer time for the resource allocation. However, in our environment, high priority message volume is significantly smaller compared to low priority message volume [10]. WXGRAPH is the main application generating $80 \%$ of the total data traffic [10] (VoIP traffic excluded) that has a service class of 10 among 12 different class of services according to [9].

In this paper, we restrict our work to scheduling algorithms running at the second level and focus on two different service classes separately. In the first class of service, we consider file transfer of graphical weather information. In the second class, we consider VoIP as an example for real-time service. Using our integrated L-DACS 1 model [13], we thoroughly analyze the performance of DRR with fragmentation scheduling for each service class in terms of delay and bandwidth fairness.

\section{L-DACS 1}

Within ICAO, currently two candidate radio access technologies for the future provision of ATS and AOS services in the L-band are considered. ${ }^{1}$ These technologies are referred to as L-DACS 1 and L-DACS 2. Initial specifications for both technologies have been published by EUROCONTROL [14], and are currently under evaluation by Single European Sky ATM Research (SESAR) project [15]. It is planned that one of these two technologies will become operational around 2025. L-DACS 1 has been designed for the transmission of both digital voice and data that provides data rates around $270 \mathrm{kbit} \mathrm{s}^{-1}$ on the Return Link (RL) and $310 \mathrm{kbit} \mathrm{s}^{-1}$ on the FL with lowest modulation and coding which we consider as baseline in our simulations. In L-DACS 1, RL and FL are separated by means of Frequency Division Duplex (FDD). In the RL, a combination of Orthogonal Frequency Division Multiple Access (OFDMA) and Time Division Multiple Access (TDMA) is used, whereas in the FL, OFDM is applied. The TDMA component in the RL is selected in order to minimize the possibility of interference with legacy systems which are operating in the L-band (e.g., the distance measuring equipment). The L-DACS 1 transmitter operates close to other receivers on board, so it should only be active for a short time, reducing these receivers' exposure to interference.

\section{A. Frame Structure}

The frame structure is shown in Fig. 1. Time is divided into superframes (SFs) with a duration of $240 \mathrm{~ms}$. At the beginning of each SF, a/c have the opportunity to $\log$ onto the network in a Random Access Channel (RACH), whereas the Base Station (BS) transmits general cell information in the Broadcast Channel (BCCH). The rest of the SF consists of four multiframes (MFs) and each MF has a duration $58.32 \mathrm{~ms}$. Each MF consists of data frames and control frames. In the FL, BS

\footnotetext{
${ }^{1}$ see http://www.eurocontrol.int/communications/
}

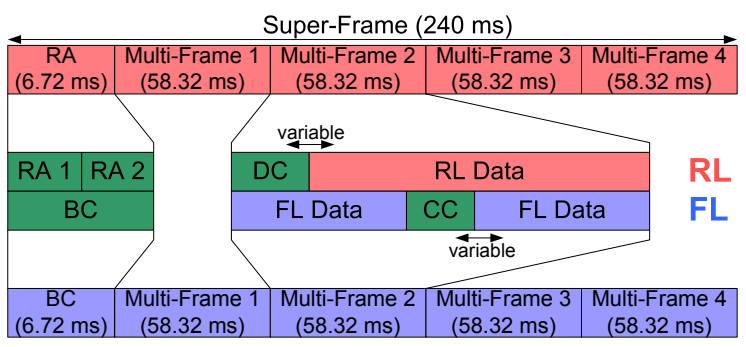

Fig. 1. L-DACS 1 frame structure

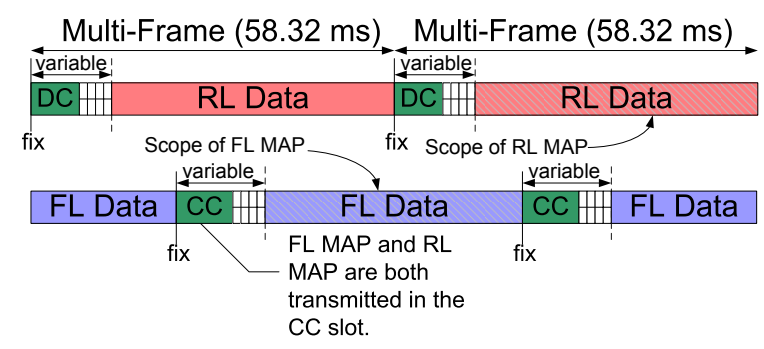

Fig. 2. L-DACS 1 resource allocation structure

transmits control information, such as resource allocations (i.e., FL and RL mapping) and acknowledgments in the Common Control Channel $(\mathrm{CCCH})$. In the RL, each $\mathrm{a} / \mathrm{c}$ is assigned one slot within the Dedicated Control Channel (DCCH) per MF for the transmission of control data. At most, $52 \mathrm{a} / \mathrm{c}$ can be accommodated within the DCCH. If more than $52 \mathrm{a} / \mathrm{c}$ are registered with a single $\mathrm{BS}$, not all of the $\mathrm{a} / \mathrm{c}$ will receive a DCCH slot in every MF. Both the $\mathrm{CCCH}$ and DCCH are of variable length in order to allow more efficient use of the wireless resources.

\section{B. Resource Allocation}

Before any transmission can take place, either in the RL or the FL, resources must be requested from the BS. At the beginning of a $\mathrm{CCCH}$ slot, the BS considers all resource requests that have been received since the last CCCH slot via RSRC_RQST message and allocates resources in terms of TDMA slots and OFDMA subchannels for the a/c and informs the a/c via RSRC_RESP message. The exact scheduling algorithm to be used by the BS is left open by the L-DACS 1 specification. In this paper, DRR with fragmentation is considered as a baseline scheduling algorithm. The allocation of resources is broadcast to all $\mathrm{a} / \mathrm{c}$ in the $\mathrm{CCCH}$ slot, specifying which $\mathrm{a} / \mathrm{c}$ is allowed to transmit when and on which subchannels on the RL and when the BS will transmit to which a/c on the FL. The scope of this resource allocation is shown in Fig. 2.

\section{QoS Mapping}

QoS mapping between applications and link technology service classes is one of the important consideration for the scheduling mechanism. In our case, on the one side L-DACS 1 supports eight different service classes and on the other side, twelve different application level class of services are defined [9]. The mapping between application CoS and L-DACS 1 


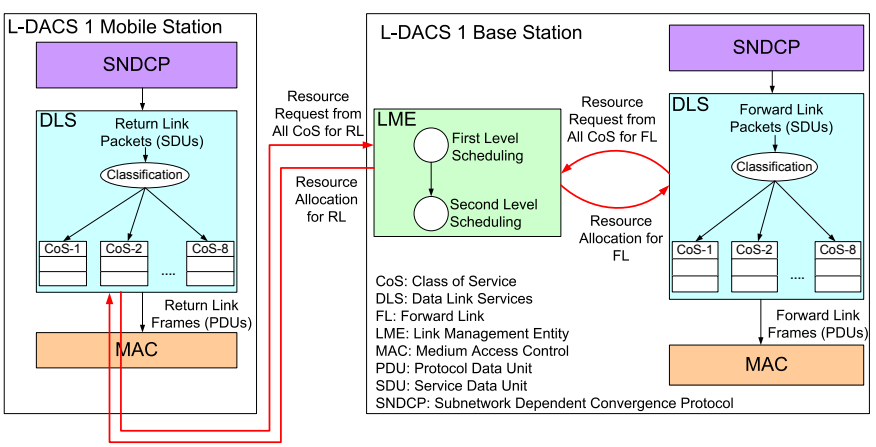

Fig. 3. L-DACS 1 link scheduling architecture

service classes are outside the scope of this paper. In this paper, we assume two different application CoSs mapped to two different L-DACS 1 service classes.

\section{Scheduling Architecture}

Fig. 3 shows the main L-DACS 1 scheduling architecture for the forward and return link in L-DACS 1 . The scheduling algorithm for forward and return link is running in L-DACS 1 BS Link Management Entity (LME) block and the packets are queued in Data Link Service (DLS) block. In the RL, L-DACS 1 only supports one type of bandwidth request mechanism: explicit contention-free bandwidth request and response. So that each a/c explicitly sends its bandwidth request in DCCH slot in order to receive resources for the RL transmission to the BS in the upcoming data slot (i.e. RL Data). After BS receives the bandwidth requests of $a / c$, the scheduling algorithm distributes the resources that correspond to the data slots of an upcoming MF (i.e. duration of $58.32 \mathrm{~ms}$ ) for both links (i.e. RL and FL) as shown in Fig. 2.

\section{DRR MODIFICATIONS}

The original DRR algorithm is proposed by [4] and the modified version which supports fragmentation is proposed by [6] ${ }^{2}$. According to [4], when a packet arrives to the queues, its flow number is checked first. In case the flow number is in the list (i.e. activelist), the packet is just enqueued corresponding to specified flow number. In case the flow is not in the list, flow number is inserted in the active list and the packet is enqueued corresponding to specified flow number. In our case, when the LME module receives resource requests of all users, it performs similar operation for the insertion of the user to active list and setting Deficit Counter (DC) to zero as shown in the first part of Algorithm 1 (i.e. lines 1-6).

Afterwards, dequeuing operation of packets takes place while the algorithm is running according to original algorithm. The algorithm checks whether the size of the head-of-line packet is smaller or equal to the DC. If this is the case, the algorithm performs the dequeuing operation, sends the packet, and decreases the DC by packet size. In the other case, the algorithm just increases the DC by a quantum size and does not send the packet.

\footnotetext{
${ }^{2}$ Our design also supports fragmentation as shown in Algorithm 1
}

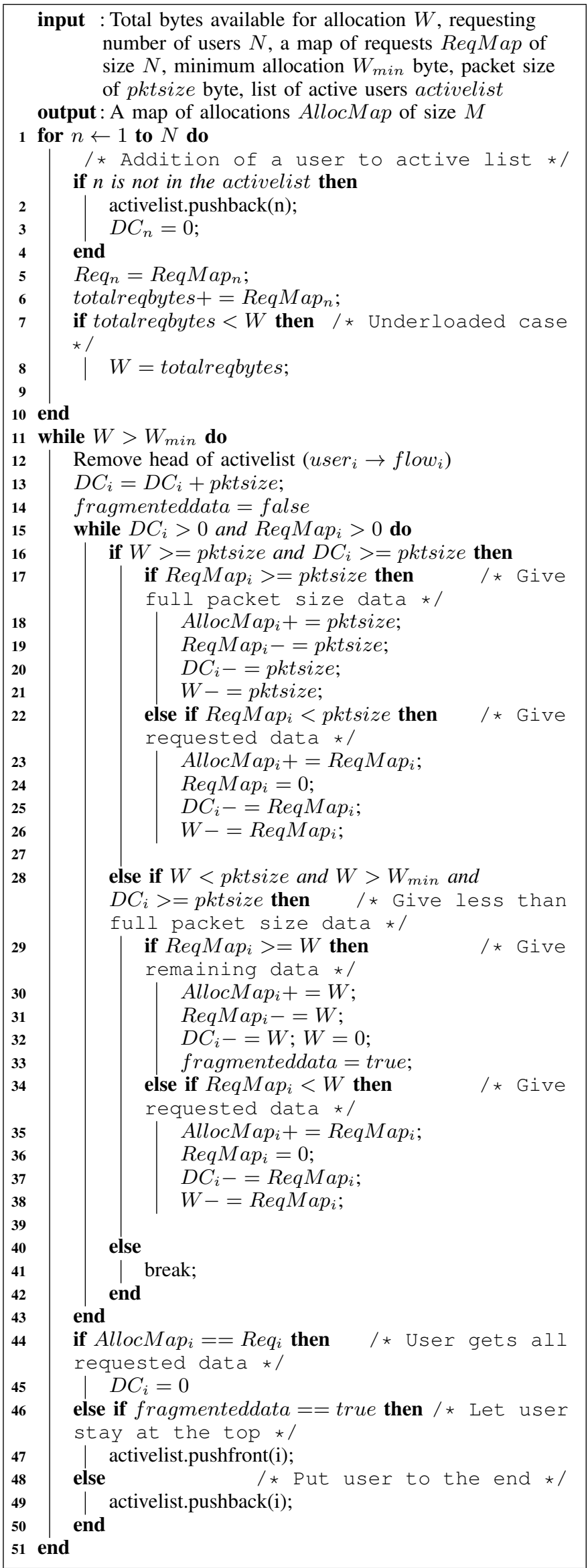

Algorithm 1: DRR with fragmentation - resource allocation in FL 
Since the original algorithm needs to know the size of the head-of-line packet, it requires certain modification for L-DACS 1. The reason is that here the queues and the scheduling are located in separate compartments. In L-DACS 1, normally in every MF, the DLS entity where the queues are located sends bandwidth requests for every user to the LME entity where the scheduling algorithm is running. For this reason, DRR is modified in a way that it operates on the bandwidth request of each user (sent by DLS) and not based on the size of the head-of-line packet as shown in the second part of the Algorithm 1 (i.e. lines 7-47). In our implementation, we select the quantum size equal to the packet size. The modified algorithm is able to run for both links (i.e., FL and RL) separately.

Due to the distributed structure of the scheduling architecture, the complexity of the scheduling process is increased as explained in the following paragraphs. There are mainly two components that increase the complexity of the scheduling process independent from the scheduling algorithm.

The first one is the transmission of resource request messages from DLS to LME, which will be processed by the scheduling algorithm. Here, if we consider $N$ number of users requesting resources then the preparation of the resource request message takes $O(N)$ complexity since we have to go through resource requests of all users.

The second one is the dequeuing operation when the scheduling algorithm running in LME gives the output of resource allocations to the DLS. Here if only $M$ number of users (out of $N$ number of requests) receive allocations according to the result of the scheduling algorithm, then the dequeuing operation should include search of packets of $M$ number of users in the queue. For each user, we have to search its packets in DLS queue where $N$ user data is located and this search adds $O(\log N)$ complexity for each user data (i.e. in our implementation we use C++ STL multimap and call find function which is $O(\log N)$ complexity). So the total complexity for $M$ number of users is $O(M) \times O(\log N)$ where $M \leq N$. In case $M \ll N$ (this is the case when quantum size (i.e. packet size) is large so that only very small amount of users receive allocation), then the total complexity becomes $\max (O(M), O(\log N))$. In case $M \sim N$ (this is the case when quantum size (i.e. packet size) is small so that almost all users receive allocation) then the complexity becomes $O(N \log N)$.

It also needs to be mentioned that the quantum size is selected in terms of bytes on the FL and in terms of protocol datagram units (PDUs) on the RL according to the bandwidth requests from DLS in BS and DLS in a/c, respectively ${ }^{3}$. For real-time services (e.g., VoIP), the quantum size is selected as $103 \mathrm{~B}$ on the FL and 8 PDUs (which makes $112 \mathrm{~B}$ for lowest coding modulation scheme) on the RL. For file transfer on the FL, we select a quantum size of $1091 \mathrm{~B}$. Because the resource requests of different CoSs are sent separately, it is possible to differentiate which quantum size is used for which CoS traffic.

\footnotetext{
${ }^{3}$ Algorithm 1 shows only resource allocation for the FL and works over in terms of bytes
}

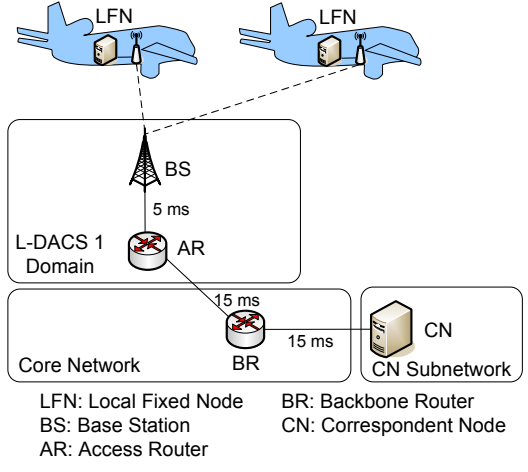

Fig. 4. Example topology for the European scenario

TABLE I

SIMULATION PARAMETERS

\begin{tabular}{ll}
\hline Parameter & Value \\
\hline Number of users (underloaded) & Forward: 15, 20; Return: 15 \\
Number of users (overloaded) & Forward: 25; Return: 20 \\
\hline TCP algorithm & Reno \\
Maximum Segment Size & $1024 \mathrm{~B}$ \\
Receiver Advertised Window & $14 \mathrm{kB}$ \\
Slow Start Threshold & $64 \mathrm{kB}$ \\
\hline
\end{tabular}

\section{Performance Evaluation}

\section{A. Assumptions}

Similar to [7], we assume that the packets are sent over ideal wireless channel conditions, so that no packets are dropped due to wireless channel impairments. We also consider finite drop-tail First-in-First-out (FIFO) queues at the BS and the mobile. For VoIP analysis, we consider buffer size of 5 packets per user in FL and 5 packets in RL. For the file transfer (i.e., the TCP analysis), we consider buffer size of 15 packets (each packet has $1 \mathrm{kB}$ ) per user in FL, which is slightly larger than the advertised receiver window size $(14 \mathrm{kB})$. Since L-DACS 1 has a contention free access scheme, we also consider overload for the VoIP scenario in order to analyze the delay fairness of the scheduling algorithm. We select smaller buffer size for VoIP traffic due to its delay sensitive characteristic.

\section{B. Considered Topology and Simulation Parameters}

The main topological considerations for the ATN/IPS are provided in [16]. In our analysis, the European scenario is considered where different numbers of a/c are communicating with an ATS or AOS CN. We assumed fixed delay values in the ground network as shown in Fig. 4 and modeled the protocols within the OMNeT++ simulator [17].

For real-time service analysis, we consider one minute of VoIP session since it is the highest measured value based on reference [18]. Here, we simulate different number of users, cf. Table I, for underloaded and overloaded scenarios. For each scenario, we collect the data of 5 different simulation runs. For file transfer analysis, TCP Reno variant is considered in the simulations with the settings shown in Table I. Here we consider a file transfer of WXGRAPH data on the FL [9] that 


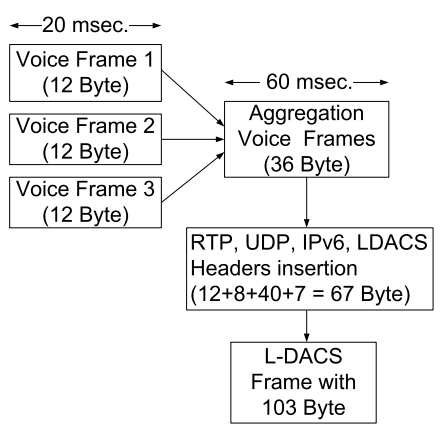

Fig. 5. Application level VoIP aggregation

requires the reliable transmission of $110 \mathrm{kB}$ of information to an a/c. Here, we simulate four different scenarios with the 5 , $10,15,20$ number of users, respectively. Similarly, for each scenario, we collect the data of 5 different simulation runs.

\section{Real-time Service Analysis}

In this section, VoIP is analyzed as an example for a realtime service. The L-DACS 1 specification already specified Advanced Multiband Excitation (AMBE) vocoder, version AMBE-ATC-10B, as a default algorithm for voice coding and decoding. The AMBE-ATC-10B vocoder produces a voice frame with 96 bit length every $20 \mathrm{~ms}$, which corresponds to a data rate of $4.8 \mathrm{kbit} \mathrm{s}^{-1}$. According to the L-DACS 1 specification, three voice frames are aggregated and transmitted together so that the overhead due to lower layers is reduced and the capacity of the wireless link is better utilized [19].

Fig. 5 shows the application layer voice frame aggregation. In addition, due to rigid timing structure of L-DACS 1, each mobile has transmit possibility only every $\mathrm{MF}$, which is around $58.32 \mathrm{~ms}$. So in case that no aggregation is used, the VoIP frames produced every $20 \mathrm{~ms}$ will enter L-DACS 1 DLS queue and wait for a transmission opportunity. The overall data rate with three frame aggregation and lower layer overhead (RTP 12 B, UDP 8 B, IPv6 40 B, L-DACS 1 $7 \mathrm{~B}$ ) becomes $13.73 \mathrm{kbit} \mathrm{s}^{-1}$. With this configuration, the total overhead due to lower layers is about $65 \%$ and almost $60 \%$ of this overhead is due to IPv6. This actually shows the need for header compression for L-DACS 1 in order to decrease the overhead to an acceptable range. However, the current specification only refers to two ITU specifications, V.42 and V.44, without providing detailed information. In this paper, we do not consider the effect of header compression in our analysis. We also consider the one-way delay attributable to codec-related processing $T_{\text {codec }}$ in IP networks as ( $N$ is denoting the number of frames) [20]:

$$
T_{\text {codec }}=(N+1) \times T_{\text {framesize }}+T_{\text {look-ahead }} .
$$

In our case, we consider $N=3$ with $T_{\text {framesize }}=20 \mathrm{~ms}$ and $T_{\text {look-ahead }}=10 \mathrm{~ms}$, thus, a total delay of $T_{\text {codec }}=90 \mathrm{~ms}$.

The ITU specifies different quality levels for voice services, where $200 \mathrm{~ms}$ one-way end-to-end delay performance is required for a "very satisfied user" [20]. As shown in Fig. 6,

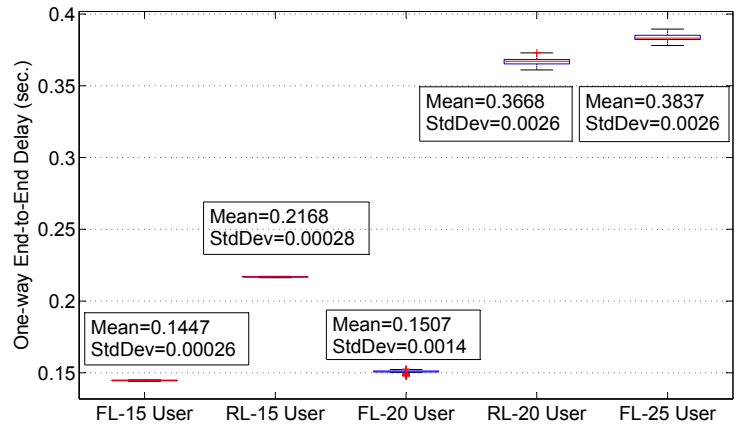

Fig. 6. Forward and return link one-way end-to-end delay

in case the system is underloaded, the average one-way delay stays almost constant. The difference between 15 users and 20 users in the FL is around $6 \mathrm{~ms}$. In this case, the two main components of the wireless link delay are

- the delay between the packet arrival time to DLS queue and service time for the packet in the next upcoming data frame, and

- the transmission delay based on the transmission speed (around $310 \mathrm{kbit} \mathrm{s}^{-1}$ on the FL is considered).

In the underloaded case, FL delay values are in the range of "very satisfied user" according to the ITU specification. However, on the RL delay values are around $0.21 \mathrm{~s}$ and correspond to the range of "satisfied user". The main reason for this result is the bandwidth request mechanism. In case the cell in not highly populated in terms of number of a/c (i.e., the number of a/c is less than 52), each user has a bandwidth request slot in every $\mathrm{DCCH}$ slot that causes an additional delay of around $60 \mathrm{~ms}$, including the time for the reception of bandwidth response in the next $\mathrm{CCCH}$ slot. In all cases, the scheduling algorithm fairly distributes the resources, so that the standard deviation of the delay is less than $0.0014 \mathrm{~s}$ as shown in Fig. 6.

If the system is overloaded (i.e., 25 users on the FL or 20 users on the RL), we observe packet drops due to buffer overflow in the DLS queues. In addition, the delay values increase to up to $0.38 \mathrm{~s}$, which is not acceptable for VoIP traffic. In this case, the scheduling algorithm treats all users again equally so that the standard deviation of delay stays below $0.0026 \mathrm{~s}$. Since VoIP traffic is affected severely in overloaded case, it is recommended to use a call-admission control mechanism.

\section{File Transfer Analysis}

In our analysis, we used the Jain's Fairness Index (JFI) as the primary performance measure, which is calculated as [21]

$$
F_{j}=\frac{\left(\sum_{i=1}^{N} x_{i}\right)^{2}}{N \cdot \sum_{i=1}^{N} x_{i}^{2}},
$$

where $N$ is the number of active users and $x_{i}$ is the normalized throughput which is defined as 
TABLE II

FAIRNESS INDEXES FOR DIFFERENT NUMBER OF USERS

\begin{tabular}{ll}
\hline Number of Users & $F_{j}$ \\
\hline 5 & 0.9999 \\
10 & 0.9999 \\
15 & 0.9998 \\
20 & 0.9998 \\
\hline
\end{tabular}

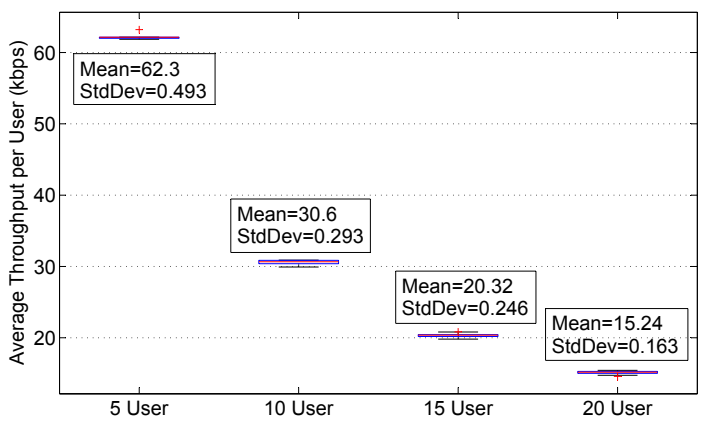

Fig. 7. Average throughput per user on the forward link

$$
x_{i}=\frac{O_{i}}{T_{i}},
$$

where $O_{i}$ is the measured throughput for each user during simulations and $T_{i}$ is the fair throughput which is the ratio of total available throughput to the number of active users. Table II shows the calculated fairness indexes for different number of users. It is clearly visible that DRR with fragmentation shows perfect fairness among all active users. In all scenarios, we did not observe any packet loss due to L-DACS 1 queue overflow as well.

Fig. 7 shows the measured average throughput of each user on the FL with different numbers of users. As expected, the average throughput decreases gradually as the number of active users increases. As the number of users increases, the scheduling algorithm continues to deliver the resources fairly among all users so that the standard deviation stays below $0.493 \mathrm{kbit} \mathrm{s}^{-1}$.

\section{CONCLUSION}

In this paper, we analyzed fairness performance of DRR with fragmentation algorithm considering two different service classes in an aeronautical networking environment. We realized that the algorithm shows very good delay and bandwidth fairness results not only in case of underloaded scenarios but also for overloaded scenarios. The need for a new bandwidth request mechanism in the RL for the VoIP service has already been recognized and input to the L-DACS 1 specification update which is currently developed within SESAR project [15]. The new design will let the mobiles request permanent allocations in the RL. We also noticed the overhead problem in the VoIP case, where the overhead reaches around $65 \%$ and mandates to use effective header compression techniques. As future work, we planned to combine different packet types (i.e. real-time and non real-time) in one DLS queue and to analyze the performance of DRR algorithm.

\section{REFERENCES}

[1] W. M. Eddy, W. Ivancic, and T. Davis, "NEMO Route Optimization Requirements for Operational Use in Aeronautics and Space Exploration Mobile Networks," IETF, RFC 5522, October 2009.

[2] "Manual for the ATN using IPS Standards and Protocols," ICAO, Tech Rep. Doc 9896-AN/469, 2009.

[3] C. So-In, R. Jain, and A.-K. Tamimi, "Scheduling in IEEE 802.16e mobile WiMAX networks: key issues and a survey," IEEE Journal on Selected Areas in Communications, vol. 27, no. 2, pp. 156-171, 2009.

[4] M. Shreedhar and G. Varghese, "Efficient fair queuing using deficit round-robin," IEEE/ACM Transactions on Networking, vol. 4, no. 3, pp. 375-385, June 1996.

[5] J. Bennett and H. Zhang, "WF2Q: worst-case fair weighted fair queueing," in IEEE INFOCOM 1997, March 1996, pp. 120-128.

[6] C. So-In, R. Jain, and A.-K. Tamimi, "A Deficit Round Robin with Fragmentation scheduler for IEEE 802.16e Mobile WiMAX," in IEEE Sarnoff Symposium, Princeton, NJ, April 2009, pp. 1-7.

[7] C. Cicconetti, A. Erta, L. Lenzini, and E. Mingozzi, "Performance Evaluation of the IEEE 802.16 MAC for QoS Support," IEEE Transactions on Mobile Computing, vol. 6, no. 1, pp. 26-38, January 2007.

[8] E. Haas, "Aeronautical channel modeling," IEEE Transactions on Vehicular Technology, vol. 51, no. 2, pp. 254-264, March 2002.

[9] "Communications Operating Concepts and Requirements for the Future Radio System," Eurocontrol/FAA, Tech. Rep., May 2007.

[10] M. Ehammer, T. Graupl, and C.-H. Rokitansky, "TCP/IP over aeronautical communication systems - Effects on bandwidth consumption," in IEEE/AIAA DASC'09, Orlando, FL, October 2009, pp. 4.A.3-1-4.A.3-9.

[11] S. Subramanya, X. Wu, H. Schulzrinne, and S. Buriak, "VoIP-based air traffic controller training," IEEE Communications Magazine, vol. 47, no. 11, pp. 148-155, November 2009.

[12] K. Wongthavarawat and A. Ganz, "Packet scheduling for QoS support in IEEE 802.16 broadband wireless access systems," International Journal of Communication Systems, vol. 16, no. 1, pp. 81-96, 2003.

[13] S. Ayaz, F. Hoffmann, C. Sommer, R. German, and F. Dressler, "Performance Evaluation of Network Mobility Handover over Future Aeronautical Data Link," in IEEE Globecom 2010, Miami, Dec. 2010.

[14] "L-DACS1 System Definition Proposal: Deliverable D2," Eurocontrol, Tech. Rep., February 2009.

[15] "WP15 - Non Avionic CNS System," SESAR Joint Undertaking, Description of Work, December 2008.

[16] C. Bauer and S. Ayaz, "ATN Topology Considerations for Aeronautical NEMO RO," IETF, Internet-Draft (work in progress) draft-bauer-mextaero-topology-01.txt, September 2009.

[17] A. Varga, "The OMNeT++ Discrete Event Simulation System," in European Simulation Multiconference (ESM 2001), Prague, Czech Republic, June 2001.

[18] N. Fistas and J. Micallef, "An Investigation into the Aircraft VHF Voice Transmissions (Aircraft DSB-AM Voice Usage Profile)," Eurocontrol, Tech. Rep., April 2004.

[19] K. Pentikousis, E. Piri, J. Pinola, F. Fitzek, T. Nissilä, and I. Harjula, "Empirical evaluation of VoIP aggregation over a fixed WiMAX testbed," in TridentCom'08. Innsbruck, Austria: ICST, 2008, pp. 19:1-19:10.

[20] "General Recommendations on the transmission quality for an entire international telephone connection,” ITU, Recommend. G.114, May 2003.

[21] R. Jain, D. Chiu, and W. Hawe, "A Quantitative Measure Of Fairness And Discrimination For Resource Allocation In Shared Computer Systems,' DEC, Research Report TR-301, September 1984. 